\title{
Light-time computations for the BepiColombo Radio Science Experiment
}

\author{
G. Tommei ${ }^{1}$, A. Milani ${ }^{1}$ and D. Vokrouhlický ${ }^{2}$ \\ ${ }^{1}$ Department of Mathematics, University of Pisa, \\ Largo B. Pontecorvo 5, 56127 Pisa, Italy \\ e-mail: tommei@dm.unipi.it, milani@dm.unipi.it \\ ${ }^{2}$ Institute of Astronomy, Charles University, \\ V Holešovičkách 2, CZ-18000 Prague 8, Czech Republic \\ e-mail: vokrouhl@cesnet.cz
}

\begin{abstract}
The Radio Science Experiment is one of the on board experiments of the Mercury ESA mission BepiColombo that will be launched in 2014 . The goals of the experiment are to determine the gravity field of Mercury and its rotation state, to determine the orbit of Mercury, to constrain the possible theories of gravitation (for example by determining the post-Newtonian $(\mathrm{PN})$ parameters), to provide the spacecraft position for geodesy experiments and to contribute to planetary ephemerides improvement. This is possible thanks to a new technology which allows to reach great accuracies in the observables range and range rate; it is well known that a similar level of accuracy requires studying a suitable model taking into account numerous relativistic effects. In this paper we deal with the modelling of the space-time coordinate transformations needed for the light-time computations and the numerical methods adopted to avoid rounding-off errors in such computations.
\end{abstract}

Keywords: Mercury, Interplanetary tracking, Light-time, Relativistic effects, Numerical methods

\section{Introduction}

BepiColombo is an European Space Agency mission to be launched in 2014, with the goal of an in-depth exploration of the planet Mercury; it has been identified as one of the most challenging long-term planetary projects. Only two NASA missions had Mercury as target in the past, the Mariner 10, which flew by three times in 1974-5 and Messenger, which carried out its flybys on January and October 2008, September 2009 before it starts its year-long orbiter phase in March 2011. 
The BepiColombo mission is composed by two spacecraft to be put in orbit around Mercury. The Radio Science Experiment is one of the on board experiments, which would coordinate a gravimetry, a rotation and a relativity experiment, using a very accurate range and range rate tracking. These measurements will be performed by a full 5-way link to the Mercury orbiter; by exploiting the frequency dependence of the refraction index, the differences between the Doppler measurements (done in Ka and $\mathrm{X}$ band) and the delay give information on the plasma content along the radio wave path (Iess and Boscagli 2001). In this way most of the measurements errors introduced can be reduced by about two orders of magnitude with respect to the past technologies. The accuracies that can be achieved are $10 \mathrm{~cm}$ in range and $3 \times 10^{-4} \mathrm{~cm} / \mathrm{s}$ in range rate.

How do we compute these observables? For example, a first approximation of the range could be given by the formula

$$
r=|\mathbf{r}|=\left|\left(\mathbf{x}_{\mathrm{sat}}+\mathbf{x}_{\mathrm{M}}\right)-\left(\mathbf{x}_{\mathrm{EM}}+\mathbf{x}_{\mathrm{E}}+\mathbf{x}_{\mathrm{ant}}\right)\right|
$$

which models a very simple geometrical situation (Figure 1). The vector $\mathbf{x}_{\text {sat }}$ is the mercurycentric position of the orbiter, the vector $\mathbf{x}_{\mathrm{M}}$ is the position of the center of mass of Mercury (M) in a reference system with origin at the Solar System Barycenter (SSB), the vector $\mathbf{x}_{\mathrm{EM}}$ is the position of the Earth-Moon center of mass in the same reference system, $\mathbf{x}_{\mathrm{E}}$ is the vector from the EarthMoon Barycenter (EMB) to the center of mass of the Earth (E), the vector $\mathbf{x}_{\text {ant }}$ is the position of the reference point of the ground antenna with respect to the center of mass of the Earth.

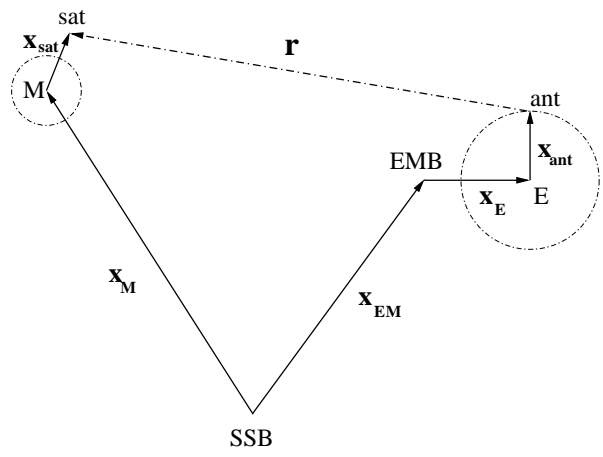

Figure 1: Geometric sketch of the vectors involved in the computation of the range. SSB is the Solar System Barycenter, M is the center of Mercury, EMB is the Earth-Moon Barycenter, $\mathrm{E}$ is the center of the Earth.

Using (1) means to model the space as a flat arena ( $r$ is an Euclidean distance) and the time as an absolute parameter. This is obviously not possible because it is clear that, beyond some threshold of accuracy, space and time have to be formulated within the framework of Einstein's theory of gravity (general relativity theory, GRT). Moreover we have to take into account the different times at which the events have to be computed: the transmission of the signal 
at the transmit time $\left(t_{t}\right)$, the signal at the Mercury orbiter at the time of bounce $\left(t_{b}\right)$ and the reception of the signal at the receive time $\left(t_{r}\right)$.

Formula (1) is used as a starting point to construct a correct relativistic formulation; with the word "correct" we do not mean all the possible relativistic effects, but the effects that can be measured by the experiment. This paper deals with the corrections to apply to this formula to obtain a consistent relativistic model for the computations of the observables and the practical implementation of such computations.

In Section 2 we discuss the relativistic four-dimensional reference systems used and the transformations adopted to make the sums in (1) consistent; according to (Soffel et al. 2003), with "reference system" we mean a purely mathematical construction, while a "reference frame" is a some physical realization of a reference system. The relativistic contribution to the time delay due to the Sun's gravitational field, the Shapiro effect, is described in Section 3. Section 4 deals with the theoretical procedure to compute the light-time (range) and the Doppler shift (range rate). In Section 5 we discuss the practical implementation of the algorithms showing how we solve the rounding-off problems.

The equations of motion for the planets Mercury and Earth, including all the relativistic effects (and potential violations of GRT) required to the accuracy of the BepiColombo Radio Science Experiment have already been discussed in (Milani et al. 2010), thus this paper focuses on the computation of the observables.

\section{Space-time reference frames and transforma- tions}

The five vectors involved in formula (1) have to be computed at their own time, the epoch of different events: e.g., $\mathbf{x}_{\mathrm{ant}}, \mathbf{x}_{\mathrm{EM}}$ and $\mathbf{x}_{\mathrm{E}}$ are computed at both the antenna transmit time $t_{t}$ and receive time $t_{r}$ of the signal. $\mathbf{x}_{\mathrm{M}}$ and $\mathbf{x}_{\text {sat }}$ are computed at the bounce time $t_{b}$ (when the signal has arrived to the orbiter and is sent back, with correction for the delay of the transponder). In order to be able to perform the vector sums and differences, these vectors have to be converted to a common space-time reference system, the only possible choice being some realization of the BCRS (Barycentric Celestial Reference System). We adopt for now a realization of the BCRS that we call SSB (Solar System Barycentric) reference frame and in which the time is a re-definition of the TDB (Barycentric Dynamic Time), according to the IAU 2006 Resolution B $3^{1}$; other possible choices, such as TCB (Barycentric Coordinate Time), only can differ by linear scaling. The TDB choice of the SSB time scale entails also the appropriate linear scaling of space-coordinates and planetary masses as described for instance in (Klioner 2008) or (Klioner et al. 2010).

The vectors $\mathbf{x}_{\mathrm{M}}, \mathbf{x}_{\mathrm{E}}$, and $\mathbf{x}_{\mathrm{EM}}$ are already in $\mathrm{SSB}$ as provided by numerical integration and external ephemerides; thus the vectors $\mathbf{x}_{\text {ant }}$ and $\mathbf{x}_{\text {sat }}$ have to

\footnotetext{
${ }^{1}$ See the Resolution at http://www.iau.org/administration/resolutions/ga2006/
} 
be converted to SSB from the geocentric and mercurycentric systems, respectively. Of course the conversion of reference system implies also the conversion of the time coordinate. There are three different time coordinates to be considered. The currently published planetary ephemerides are provided in TDB. The observations are based on averages of clock and frequency measurements on the Earth surface: this defines another time coordinate called TT (Terrestrial Time). Thus for each observation the times of transmission $t_{t}$ and reception $t_{r}$ need to be converted from TT to TDB to find the corresponding positions of the planets, e.g., the Earth and the Moon, by combining information from the pre-computed ephemerides and the output of the numerical integration for Mercury and for the Earth-Moon barycenter. This time conversion step is necessary for the accurate processing of each set of interplanetary tracking data; the main term in the difference TT-TDB is periodic, with period 1 year and amplitude $\simeq 1.6 \times 10^{-3} \mathrm{~s}$, while there is essentially no linear trend, as a result of a suitable definition of the TDB.

The equation of motion of a mercurycentric orbiter can be approximated, to the required level of accuracy, by a Newtonian equation provided the independent variable is the proper time of Mercury. Thus, for the BepiColombo Radio Science Experiment, it is necessary to define a new time coordinate TDM (Mercury Dynamic Time), as described in (Milani et al. 2010), containing terms of 1-PN order depending mostly upon the distance from the Sun and velocity of Mercury.

From now on, in accordance with (Klioner et al. 2010), we shall call the quantities related to the SSB frame "TDB-compatible", the quantities related to the geocentric frame "TT-compatible", the quantities related to the mercurycentric frame "TDM-compatible" and label them TB, TT and TM, respectively.

The differential equation giving the local time $T$ as a function of the SSB time $t$, which we are currently assuming to be TDB, is the following:

$$
\frac{d T}{d t}=1-\frac{1}{c^{2}}\left[U+\frac{v^{2}}{2}-L\right],
$$

where $U$ is the gravitational potential (the list of contributing bodies depends upon the accuracy required: in our implementation we use Sun, Mercury to Neptune, Moon) at the planet center and $v$ is the SSB velocity of the same planet. The constant term $L$ is used to perform the conventional rescaling motivated by removal of secular terms, e.g., for the Earth we use $L_{C}$ (Soffel et al. 2003).

The space-time transformations to perform involve essentially the position of the antenna and the position of the orbiter. The geocentric coordinates of the antenna should be transformed into TDB-compatible coordinates; the transformation is expressed by the formula

$$
\mathbf{x}_{\mathrm{ant}}^{T B}=\mathbf{x}_{\mathrm{ant}}^{T T}\left(1-\frac{U}{c^{2}}-L_{C}\right)-\frac{1}{2}\left(\frac{\mathbf{v}_{\mathrm{E}}^{T B} \cdot \mathbf{x}_{\mathrm{ant}}^{T T}}{c^{2}}\right) \mathbf{v}_{\mathrm{E}}^{T B},
$$

where $U$ is the gravitational potential at the geocenter (excluding the Earth mass), $L_{C}=1.48082686741 \times 10^{-8}$ is a scaling factor given as definition, sup- 
posed to be a good approximation for removing secular terms from the transformation and $\mathbf{v}_{\mathrm{E}}^{T B}$ is the barycentric velocity of the Earth. The next formula contains the effect on the velocities of the time coordinate change, which should be consistently used together with the coordinate change:

$$
\mathbf{v}_{\text {ant }}^{T B}=\left[\mathbf{v}_{\text {ant }}^{T T}\left(1-\frac{U}{c^{2}}-L_{C}\right)-\frac{1}{2}\left(\frac{\mathbf{v}_{\mathrm{E}}^{T B} \cdot \mathbf{v}_{\mathrm{ant}}^{T T}}{c^{2}}\right) \mathbf{v}_{\mathrm{E}}^{T B}\right]\left[\frac{d T}{d t}\right] .
$$

Note that the previous formula contains the factor $d T / d t$ (expressed by (2)) that deals with a time transformation: $T$ is the local time for Earth, that is TT, and $t$ is the corresponding TDB time.

The mercurycentric coordinates of the orbiter have to be transformed into TDB-compatible coordinates through the formula

$$
\mathbf{x}_{\mathrm{sat}}^{T B}=\mathbf{x}_{\mathrm{sat}}^{T M}\left(1-\frac{U}{c^{2}}-L_{C M}\right)-\frac{1}{2}\left(\frac{\mathbf{v}_{\mathrm{M}}^{T B} \cdot \mathbf{x}_{\mathrm{sat}}^{T M}}{c^{2}}\right) \mathbf{v}_{\mathrm{M}}^{T B},
$$

where $U$ is the gravitational potential at the center of mass of Mercury (excluding the Mercury mass) and $L_{C M}$ could be used to remove the secular term in the time transformation (thus defining a TM scale, implying a rescaling of the mass of Mercury). We believe this is not necessary: the secular drift of TDM with respect to other time scales is significant, see Figure 5 in (Milani et al. 2010), but a simple iterative scheme is very efficient in providing the inverse time transformation. Thus we set $L_{C M}=0$, assuming the reference frame is TDM-compatible. As for the antenna we have a formula expressing the velocity transformation that contains the derivative of time $T$ for Mercury, that is TDM, with respect to time $t$, that is TDB:

$$
\mathbf{v}_{\mathrm{sat}}^{T B}=\left[\mathbf{v}_{\mathrm{sat}}^{T M}\left(1-\frac{U}{c^{2}}-L_{C M}\right)-\frac{1}{2}\left(\frac{\mathbf{v}_{\mathrm{M}}^{T B} \cdot \mathbf{v}_{\mathrm{sat}}^{T M}}{c^{2}}\right) \mathbf{v}_{\mathrm{M}}^{T B}\right]\left[\frac{d T}{d t}\right] .
$$

For these coordinate changes, in every formula we neglected the terms of the SSB acceleration of the planet center (Damour et al. 1994), because they contain beside $1 / c^{2}$ the additional small parameter (distance from planet center) / (planet distance to the Sun), which is of the order of $10^{-4}$ even for a Mercury orbiter.

To assess the relevance of the relativistic corrections of this section to the accuracy of the BepiColombo Radio Science Experiment, we computed the observables range and range rate with and without these corrections. As shown in Figure 2, the differences are significant, at a signal-to-noise ratio $S / N \simeq 1$ for range, much more for range rate, with an especially strong signature from the orbital velocity of the mercurycentric orbit (with $S / N>50$ ).

\section{Shapiro effect}

The correct modelling of space-time transformations is not sufficient to have a precise computation of the signal delay: we have to take into account the general relativistic contribution to the time delay due to the space-time curvature under 

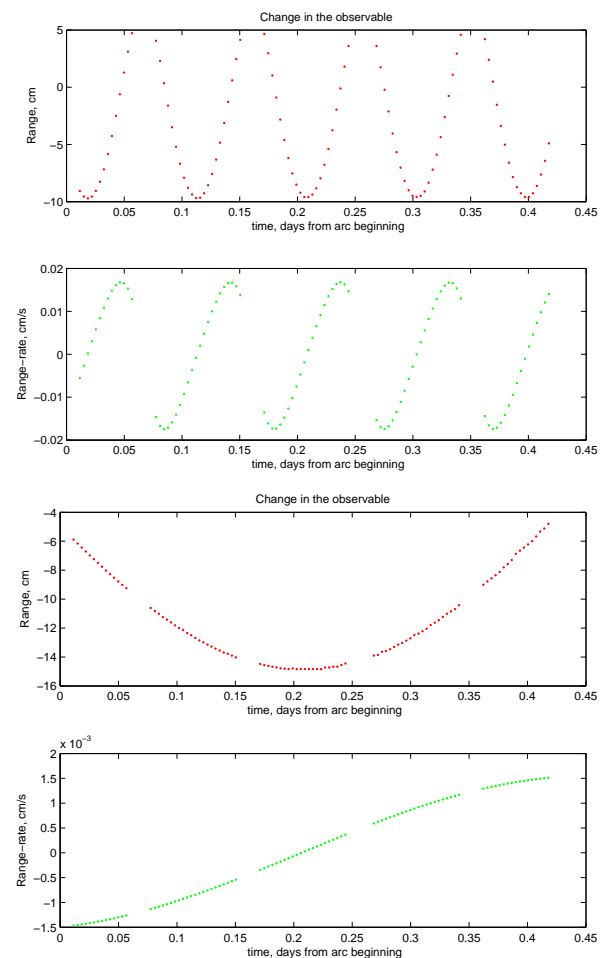

Figure 2: The difference in the observables range and range rate for one pass of Mercury above the horizon for a ground station, by using an hybrid model in which the position and velocity of the orbiter have not transformed to TDB-compatible quantities and a correct model in which all quantities are TDB-compatible. Interruptions of the signal are due to spacecraft passage behind Mercury as seen for the Earth station. Top: for an hybrid model with the satellite position and velocity not transformed to TDB-compatible. Bottom: for an hybrid model with the position and velocity of the antenna not transformed to TDB-compatible.

the effect of the Sun's gravitational field, the Shapiro effect (Shapiro 1964). The Shapiro time delay $\Delta t$ at the 1-PN level, according to (Will 1993) and (Moyer 2003), is

$$
\Delta t=\frac{(1+\gamma) \mu_{0}}{c^{3}} \ln \left(\frac{r_{t}+r_{r}+r}{r_{t}+r_{r}-r}\right), \quad S(\gamma)=c \Delta t
$$

where $r_{t}=\left|\mathbf{r}_{\mathrm{t}}\right|$ and $r_{r}=\left|\mathbf{r}_{\mathrm{r}}\right|$ are the heliocentric distances of the transmitter and the receiver at the corresponding time instants of photon transmission and reception, $\mu_{0}$ is the gravitational mass of the Sun $\left(\mu_{0}=G m_{0}\right)$ and $r=\left|\mathbf{r}_{r}-\mathbf{r}_{t}\right|$. The planetary terms, similar to the solar one, can also be included but they are smaller than the accuracy needed for our measurements. Parameter $\gamma$ is the only post-Newtonian parameter used for the light-time effect and, in fact, it could be best constrained during superior conjunction (Milani et al. 2002). 
The total amount of the Shapiro effect in range is shown in Figure 3.

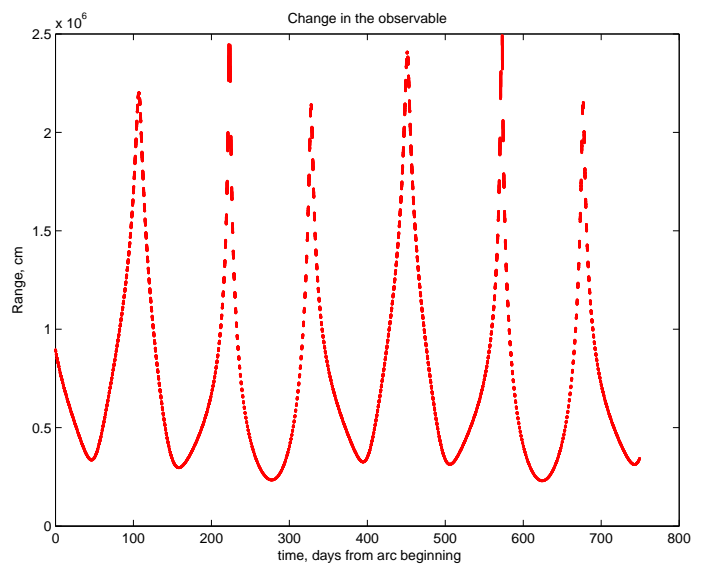

Figure 3: Total amount of the Shapiro effect in range over 2-year simulation. The sharp peaks correspond to superior conjunctions, when Mercury is "behind the Sun" as seen from Earth, with values as large as $24 \mathrm{~km}$ for radio waves passing at 3 solar radii from the center of the Sun. Interruptions of the signal are due to spacecraft visibility from the Earth station (in this simulation we assume just one station).

The question arises whether the very high signal to noise in the range requires other terms in the solar gravity influence, due to either (i) motion of the source, or (ii) higher-order corrections when the radio waves are passing near the Sun, at just a few solar radii (and thus the denominator in the log-function of the Shapiro formula is small). The corrections (i) are of the post-Newtonian order 1.5 (containing a factor $1 / c^{3}$ ), but it has been shown in (Milani et al. 2010) that they are too small to affect our accuracy. The corrections (ii) are of order 2 , (containing a factor $1 / c^{4}$ ), but they can be actually larger for an experiment involving Mercury. The relevant correction is most easily obtained by adding $1 / c^{4}$ terms in the Shapiro formula, due to the bending of the light path:

$$
S(\gamma)=\frac{(1+\gamma) \mu_{0}}{c^{2}} \ln \left(\frac{r_{t}+r_{r}+r+\frac{(1+\gamma) \mu_{0}}{c^{2}}}{r_{t}+r_{r}-r+\frac{(1+\gamma) \mu_{0}}{c^{2}}}\right) .
$$

This formulation has been proposed by (Moyer 2003) and it has been justified in the small impact parameter regime by much more theoretically rooted derivations by (Klioner and Zschocke 2007), (Teyssandier and Le Poncin-Lafitte 2008) and (Ashby and Bertotti 2009). Figure 4 shows that the order 2 correction is relevant for our experiment, especially when there is a superior conjunction with a small impact parameter of the radio wave path passing near the Sun. In practice there is a lower bound to the impact parameter because of the turbulence of the solar corona: below 10 solar radii the measurement accuracy is degraded, thus this effect is marginally significant, but is not entirely negligible. Note 
that the $1 / c^{4}$ correction $(\sim 10 \mathrm{~cm})$ in the Shapiro formula effectively corresponds to $\sim 3 \times 10^{-5}$ correction in the value of the post-Newtonian parameter $\gamma$. The Shapiro correction for the computation of the range rate will be shown in Section 4.
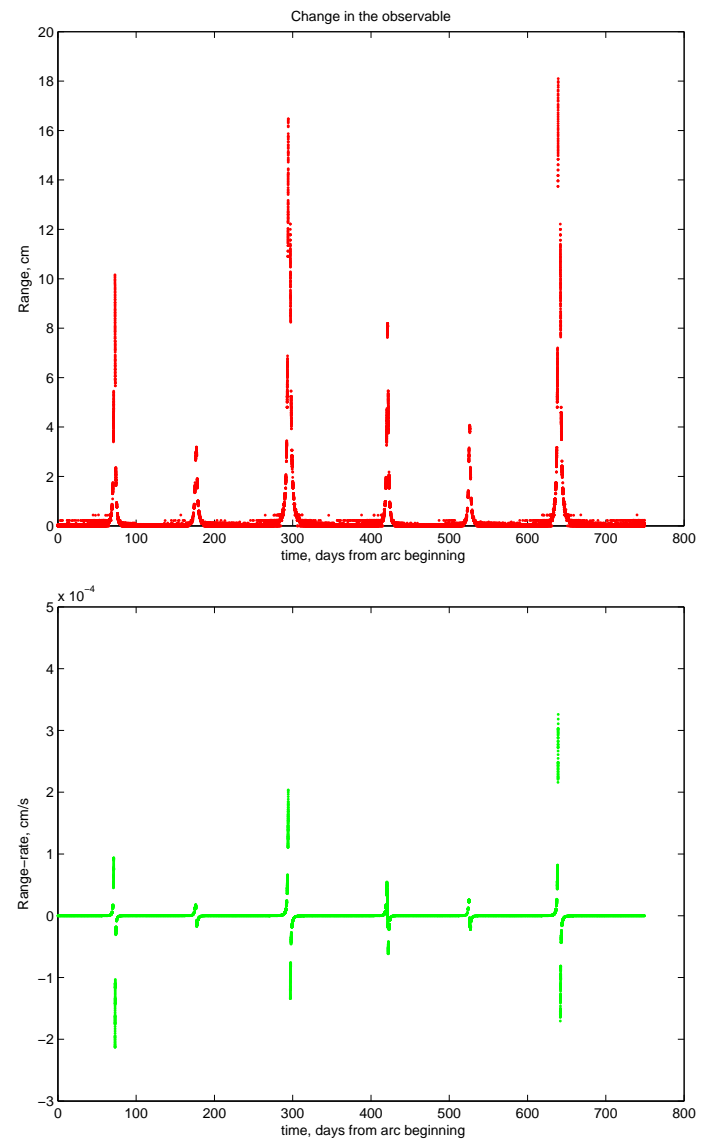

Figure 4: Differences in range (top) and range rate (bottom) by using an order 1 and an order 2 post-Newtonian formulation $(\gamma=1)$; the correction is relevant for BepiColombo, at least when a superior conjunction results in a small impact parameter $b$. E.g., in this figure we have plotted data assumed to be available down to 3 solar radii. For larger values of $b$ the effect decreases as $1 / b^{2}$.

\section{Light-time iterations}

Since radar measurements are usually referred to the receive time $t_{r}$ the observables are seen as functions of this time, and the computation sequence works backward in time: starting from $t_{r}$, the bounce time $t_{b}$ is computed iteratively, 
and, using this information the transmit time $t_{t}$ is computed.

The vectors $\mathbf{x}_{\mathrm{M}}^{T B}$ and $\mathbf{x}_{\mathrm{EM}}^{T B}$ are obtained integrating the post-Newtonian equations of motion. The vectors $\mathbf{x}_{\text {sat }}^{T M}$ are obtained by integrating the orbit in the mercurycentric TDM-compatible frame. The vector $\mathbf{x}_{\mathrm{ant}}^{T T}$ is obtained from a standard IERS model of Earth rotation, given accurate station coordinates, and $\mathbf{x}_{\mathrm{E}}^{T T}$ from lunar ephemerides (Milani and Gronchi 2010). In the following subsections we shall describe the procedure to compute the range (Section 4.1) and the range rate (Section 4.2 ).

\subsection{Range}

Once the five vectors are available at the appropriate times and in a consistent SSB system, there are two different light-times, the up-leg $\Delta t_{u p}=t_{b}-t_{t}$ for the signal from the antenna to the orbiter, and the down-leg $\Delta t_{\text {down }}=t_{r}-t_{b}$ for the return signal. They are defined implicitly by the distances down-leg and up-leg

$$
\begin{gathered}
\mathbf{r}_{d o}\left(t_{r}\right)=\mathbf{x}_{\mathrm{sat}}\left(t_{b}\left(t_{r}\right)\right)+\mathbf{x}_{\mathrm{M}}\left(t_{b}\left(t_{r}\right)\right)-\mathbf{x}_{\mathrm{EM}}\left(t_{r}\right)-\mathbf{x}_{\mathrm{E}}\left(t_{r}\right)-\mathbf{x}_{\mathrm{ant}}\left(t_{r}\right), \\
r_{d o}\left(t_{r}\right)=\left|\mathbf{r}_{d o}\left(t_{r}\right)\right|, \quad c\left(t_{r}-t_{b}\right)=r_{d o}\left(t_{r}\right)+S_{d o}(\gamma), \\
\mathbf{r}_{u p}\left(t_{r}\right)=\mathbf{x}_{\mathrm{sat}}\left(t_{b}\left(t_{r}\right)\right)+\mathbf{x}_{\mathrm{M}}\left(t_{b}\left(t_{r}\right)\right)-\mathbf{x}_{\mathrm{EM}}\left(t_{t}\left(t_{r}\right)\right)-\mathbf{x}_{\mathrm{E}}\left(t_{t}\left(t_{r}\right)\right)-\mathbf{x}_{\text {ant }}\left(t_{t}\left(t_{r}\right)\right), \\
r_{u p}\left(t_{r}\right)=\left|\mathbf{r}_{u p}\left(t_{r}\right)\right|, \quad c\left(t_{b}-t_{t}\right)=r_{u p}\left(t_{r}\right)+S_{u p}(\gamma),
\end{gathered}
$$

respectively, with somewhat different Shapiro effects:

$$
\begin{aligned}
& S_{d o}(\gamma)=\frac{(1+\gamma) \mu_{0}}{c^{2}} \ln \left(\frac{r_{t}\left(t_{b}\right)+r_{r}\left(t_{r}\right)+\left|\mathbf{r}_{r}\left(t_{r}\right)-\mathbf{r}_{t}\left(t_{b}\right)\right|+\frac{(1+\gamma) \mu_{0}}{c^{2}}}{r_{t}\left(t_{b}\right)+r_{r}\left(t_{r}\right)-\left|\mathbf{r}_{r}\left(t_{r}\right)-\mathbf{r}_{t}\left(t_{b}\right)\right|+\frac{(1+\gamma) \mu_{0}}{c^{2}}}\right), \\
& S_{u p}(\gamma)=\frac{(1+\gamma) \mu_{0}}{c^{2}} \ln \left(\frac{r_{t}\left(t_{t}\right)+r_{r}\left(t_{b}\right)+\left|\mathbf{r}_{r}\left(t_{b}\right)-\mathbf{r}_{t}\left(t_{t}\right)\right|+\frac{(1+\gamma) \mu_{0}}{c^{2}}}{r_{t}\left(t_{t}\right)+r_{r}\left(t_{b}\right)-\left|\mathbf{r}_{r}\left(t_{b}\right)-\mathbf{r}_{t}\left(t_{t}\right)\right|+\frac{(1+\gamma) \mu_{0}}{c^{2}}}\right) .
\end{aligned}
$$

Note that, for the down-leg, the vector $\mathbf{r}_{t}$ refers to the heliocentric position of the spacecraft and it is computed at the bounce time, while, for the up-leg, the same vector refers to the heliocentric position of the antenna and it is computed at the transmit time. This difference occurs also for the vector $\mathbf{r}_{r}$ : in the downleg it refers to the position of the antenna at the receive time, while in the up-leg to the position of the spacecraft at the bounce time.

Then $t_{r}-t_{b}$ and $t_{b}-t_{t}$ are the two portions of the light-time, in the time attached to the SSB, that is TDB; this provides the computation of $t_{t}$. Then these times are to be converted back in the time system applicable at the receiving station, where the time measurement is performed, which is TT (or some other form of local time, such as the standard UTC). The time $t_{r}$ is already available in the local time scale, from the original measurement, while $t_{t}$ needs to be converted back from TDB to TT. The difference between these two TT times is $\Delta t_{t o t}$, from which we can conventionally define $r\left(t_{r}\right)=c \Delta t_{t o t} / 2$. Note that 
the difference $\Delta t_{t o t}$ in TT is significantly different from $t_{r}-t_{t}$ in TDB, by an amount of the order of $10^{-7} \mathrm{~s}$, while the sensitivity of the BepiColombo Radio Science Experiment is of the order of $10^{-9} \mathrm{~s}$, thus these conversions change the computed observable in a significant way (Figure 5).
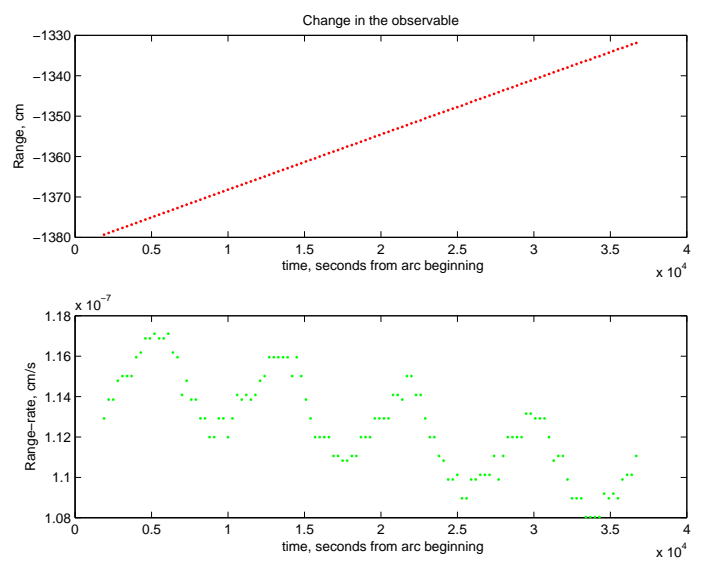

Figure 5: The difference in the observables range and range rate using a light-time in TT and a light time in TDB: the difference in range is very high, more than 13 meters in one day, while the difference in range rate is less than the accuracy of the experiment.

The practical method for solving $t_{b}\left(t_{r}\right)$ and $t_{t}\left(t_{r}\right)$ in (3) and (4) is as follows. Since the measurement is labeled with the receive time $t_{r}$, the iterative procedure needs to start from (3) by computing the states $\mathbf{x}_{\mathrm{EM}}, \mathbf{x}_{\mathrm{E}}$ and $\mathbf{x}_{\mathrm{ant}}$ at epoch $t_{r}$, then selecting a rough guess $t_{b}^{0}$ for the bounce time (e.g., $\left.t_{b}^{0}=t_{r}\right)$. Then the states $\mathbf{x}_{\mathrm{sat}}$ and $\mathbf{x}_{\mathrm{M}}$ are computed at $t_{b}^{0}$ and a successive guess $t_{b}^{1}$ is given by (3). This is repeated computing $t_{b}^{2}$, and so on until convergence, that is, until $t_{b}^{k}-t_{b}^{k-1}$ is smaller than the required accuracy. This fixed point iteration to solve the implicit equation for $t_{b}$ is convergent because the motion of the satellite and of Mercury, in the time $t_{r}-t_{b}$, is a small fraction of the total difference vector. After accepting the last value of $t_{b}$ we start with the states $\mathbf{x}_{\mathrm{sat}}$ and $\mathbf{x}_{\mathrm{M}}$ at $t_{b}$ and with a rough guess $t_{t}^{0}$ for the transmit time (e.g., $t_{t}^{0}=t_{b}$ ). Then $\mathbf{x}_{\mathrm{EM}}, \mathbf{x}_{\mathrm{E}}$ and $\mathbf{x}_{\text {ant }}$ are computed at epoch $t_{t}^{0}$ and $t_{t}^{1}$ is given by (4), and the same procedure is iterated to convergence, that is to achieve a small enough $t_{t}^{k}-t_{t}^{k-1}$. This double iterative procedure to compute range is consistent with what has been used for a long time in planetary radar, as described in (Yeomans et al. 1992). We conventionally define $r=\left(r_{d o}+S_{d o}+r_{u p}+S_{u p}\right) / 2$.

\subsection{Range rate}

After the two iterations providing at convergence $t_{b}$ and $t_{t}$ are complete, we can proceed to compute the range rate. We shall use the following notation:

- $\frac{d}{d t_{r}}$ stands for the total derivative with respect to the receive time $t_{r}$; 
- $\frac{\partial}{\partial t_{b}}$ stands for the partial derivative with respect to the receive time $t_{b}$;

- $\frac{\partial}{\partial t_{t}}$ stands for the partial derivative with respect to the receive time $t_{t}$.

We rewrite the expression for the Euclidean range (down-leg and up-leg) as a scalar product:

$$
\begin{aligned}
r_{d o}^{2}\left(t_{r}\right) & =\left[\mathbf{x}_{\mathrm{Ms}}\left(t_{b}\right)-\mathbf{x}_{\mathrm{Ea}}\left(t_{r}\right)\right] \cdot\left[\mathbf{x}_{\mathrm{Ms}}\left(t_{b}\right)-\mathbf{x}_{\mathrm{Ea}}\left(t_{r}\right)\right], \\
r_{u p}^{2}\left(t_{r}\right) & =\left[\mathbf{x}_{\mathrm{Ms}}\left(t_{b}\right)-\mathbf{x}_{\mathrm{Ea}}\left(t_{t}\right)\right] \cdot\left[\mathbf{x}_{\mathrm{Ms}}\left(t_{b}\right)-\mathbf{x}_{\mathrm{Ea}}\left(t_{t}\right)\right],
\end{aligned}
$$

where $\mathbf{x}_{\mathrm{Ms}}=\mathbf{x}_{\mathrm{M}}+\mathbf{x}_{\mathrm{sat}}$ and $\mathbf{x}_{\mathrm{Ea}}=\mathbf{x}_{\mathrm{EM}}+\mathbf{x}_{\mathrm{E}}+\mathbf{x}_{\mathrm{ant}}$. The light-time equation contains also the Shapiro terms, thus the range rate observable contains also additive terms $d S_{d o} / d t_{r}$ and $d S_{u p} / d t_{r}$, with significant effects (a few $\mathrm{cm} / \mathrm{s}$ during superior conjunctions). Since the equations giving $t_{b}$ and $t_{t}$ are still (3) and (4), in computing the time derivatives, we need to take into account that $t_{b}=t_{b}\left(t_{r}\right)$ and $t_{t}=t_{t}\left(t_{r}\right)$, with non-unit derivatives. By computing the derivative with respect to the receive time $t_{r}$ we obtain

$$
\frac{d}{d t_{r}}\left[r_{d o}\left(t_{r}\right)+S_{d o}\left(t_{r}\right)\right]=\hat{\mathbf{r}}_{d o} \cdot\left[\frac{\partial \mathbf{x}_{\mathrm{Ms}}\left(t_{b}\right)}{\partial t_{b}} \frac{d t_{b}}{d t_{r}}-\frac{d \mathbf{x}_{\mathrm{Ea}}\left(t_{r}\right)}{d t_{r}}\right]+\frac{d S_{d o}}{d t_{r}}
$$

where

$$
\hat{\mathbf{r}}_{d o}=\frac{\mathbf{x}_{\mathrm{Ms}}\left(t_{b}\right)-\mathbf{x}_{\mathrm{Ea}}\left(t_{r}\right)}{r_{d o}\left(t_{r}\right)}, \quad \frac{d t_{b}}{d t_{r}}=1-\frac{d r_{d o}\left(t_{r}\right) / d t_{r}+d S_{d o} / d t_{r}}{c}
$$

and

$$
\frac{d}{d t_{r}}\left[r_{u p}\left(t_{r}\right)+S_{u p}\left(t_{r}\right)\right]=\hat{\mathbf{r}}_{u p} \cdot\left[\frac{\partial \mathbf{x}_{\mathrm{Ms}}\left(t_{b}\right)}{\partial t_{b}} \frac{d t_{b}}{d t_{r}}-\frac{\partial \mathbf{x}_{\mathrm{Ea}}\left(t_{t}\right)}{\partial t_{t}} \frac{d t_{t}}{d t_{r}}\right]+\frac{d S_{u p}}{d t_{r}}(6)
$$

where

$$
\begin{gathered}
\hat{\mathbf{r}}_{u p}=\frac{\mathbf{x}_{\mathrm{Ms}}\left(t_{b}\right)-\mathbf{x}_{\mathrm{Ea}}\left(t_{t}\right)}{r_{u p}\left(t_{r}\right)} \\
\frac{d t_{t}}{d t_{r}}=1-\frac{d r_{d o}\left(t_{r}\right) / d t_{r}+d S_{d o} / d t_{r}}{c}-\frac{d r_{u p}\left(t_{r}\right) / d t_{r}+d S_{u p} / d t_{r}}{c}
\end{gathered}
$$

The derivatives of the Shapiro effect are

$$
\begin{aligned}
\frac{d S_{d o}}{d t_{r}}= & \frac{2(1+\gamma) \mu_{0}}{c^{2}}\left[\left(r_{t}\left(t_{b}\right)+r_{r}\left(t_{r}\right)+\frac{(1+\gamma) \mu_{0}}{c^{2}}\right)^{2}-\left|\mathbf{r}_{r}\left(t_{r}\right)-\mathbf{r}_{t}\left(t_{b}\right)\right|^{2}\right]^{-1}(7) \\
& {\left[-\left|\mathbf{r}_{r}\left(t_{r}\right)-\mathbf{r}_{t}\left(t_{b}\right)\right|\left(\frac{\partial r_{t}}{\partial t_{b}} \frac{d t_{b}}{d t_{r}}+\frac{d r_{r}}{d t_{r}}\right)+\right.} \\
& \left.\frac{\mathbf{r}_{r}\left(t_{r}\right)-\mathbf{r}_{t}\left(t_{b}\right)}{\left|\mathbf{r}_{r}\left(t_{r}\right)-\mathbf{r}_{t}\left(t_{b}\right)\right|} \cdot\left(\frac{d \mathbf{r}_{r}}{d t_{r}}-\frac{\partial \mathbf{r}_{t}}{\partial t_{b}} \frac{d t_{b}}{d t_{r}}\right)\left(r_{t}\left(t_{b}\right)+r_{r}\left(t_{r}\right)+\frac{(1+\gamma) \mu_{0}}{c^{2}}\right)\right],
\end{aligned}
$$




$$
\begin{aligned}
\frac{d S_{u p}}{d t_{r}}= & \frac{2(1+\gamma) \mu_{0}}{c^{2}}\left[\left(r_{t}\left(t_{t}\right)+r_{r}\left(t_{b}\right)+\frac{(1+\gamma) \mu_{0}}{c^{2}}\right)^{2}-\left|\mathbf{r}_{r}\left(t_{b}\right)-\mathbf{r}_{t}\left(t_{t}\right)\right|^{2}\right]^{-1} \\
& {\left[-\left|\mathbf{r}_{r}\left(t_{b}\right)-\mathbf{r}_{t}\left(t_{t}\right)\right|\left(\frac{\partial r_{t}}{\partial t_{t}} \frac{d t_{t}}{d t_{r}}+\frac{\partial r_{r}}{\partial t_{b}} \frac{d t_{b}}{d t_{r}}\right)+\right.} \\
& \left.\frac{\mathbf{r}_{r}\left(t_{b}\right)-\mathbf{r}_{t}\left(t_{t}\right)}{\left|\mathbf{r}_{r}\left(t_{r}\right)-\mathbf{r}_{t}\left(t_{b}\right)\right|} \cdot\left(\frac{\partial \mathbf{r}_{r}}{\partial t_{b}} \frac{d t_{b}}{d t_{r}}-\frac{\partial \mathbf{r}_{t}}{\partial t_{t}} \frac{d t_{t}}{d t_{r}}\right)\left(r_{t}\left(t_{t}\right)+r_{r}\left(t_{b}\right)+\frac{(1+\gamma) \mu_{0}}{c^{2}}\right)\right] .
\end{aligned}
$$

Note that, because of different definitions of $\mathbf{r}_{t}$ and $\mathbf{r}_{r}$ in the down-leg and up-leg (Section 4.1), the term $\partial r_{t} / \partial t_{b}$ in the second row of (7) is exactly the same thing as $\partial r_{r} / \partial t_{b}$ in the second row of (8). However, the contribution of the time derivatives of the Shapiro effect to the $d t_{b} / d t_{r}$ and $d t_{t} / d t_{r}$ corrective factors is small, of the order of $10^{-10}$, which is marginally significant for the BepiColombo Radio Science Experiment. We conventionally define the range rate $d r / d t_{r}=c\left(1-d t_{t} / d t_{r}\right) / 2=\left(d r_{d o} / d t_{r}+d S_{d o} / d t_{r}+d r_{u p} / d t_{r}+d S_{u p} / d t_{r}\right) / 2$. These equations are compatible with the equations in (Yeomans et al. 1992), taking into account that they use a single iteration. Equations (7) and (8) are almost never found in the literature and has not been much used in the processing of the past radio science experiments (Bertotti et al. 2003) because the observable range rate is typically computed as difference of ranges divided by time; however, for reasons explained in Section 5, these formulas are now necessary.

Since the time derivatives of the Shapiro effects contain $d t_{b} / d t_{r}$ and $d t_{t} / d t_{r}$, the equations (5) and (6) are implicit, thus we can again use a fixed point iteration. It is also possible to use a very good approximation which solves explicitly for $d r_{d o} / d t_{r}$ and then for $d r_{u p} / d t_{r}$, neglecting the very small contribution of Shapiro terms:

$\frac{d r_{d o}}{d t_{r}}=\hat{\mathbf{r}}_{d o} \cdot\left[\frac{\partial \mathbf{x}_{\mathrm{Ms}}\left(t_{b}\right)}{\partial t_{b}}\left(1-\frac{d S_{d o} / d t_{r}}{c}\right)-\frac{d \mathbf{x}_{\mathrm{Ea}}\left(t_{r}\right)}{d t_{r}}\right]\left[1+\frac{1}{c}\left(\frac{\partial \mathbf{x}_{\mathrm{Ms}}\left(t_{b}\right)}{\partial t_{b}} \cdot \hat{\mathbf{r}}_{d o}\right)\right]^{-1}$,

where the right hand side is weakly dependent upon $d r_{d o} / d t_{r}$ only through $d S_{d o} / d t_{r}$, thus a moderately accurate approximation could be used in the computation of $d S_{d o} / d t_{r}$, followed by a single iteration. For the other leg

$$
\begin{gathered}
\frac{d r_{u p}}{d t_{r}}=\hat{\mathbf{r}}_{u p} \cdot\left[\frac{\partial \mathbf{x}_{\mathrm{Ms}}\left(t_{b}\right)}{\partial t_{b}}\left(1-\frac{d r_{d o}\left(t_{r}\right) / d t_{r}+d S_{d o} / d t_{r}}{c}\right)-\right. \\
\left.\frac{\partial \mathbf{x}_{\mathrm{Ea}}\left(t_{t}\right)}{\partial t_{t}}\left(1-\frac{d r_{d o}\left(t_{r}\right) / d t_{r}+d S_{d o} / d t_{r}+d S_{u p} / d t_{r}}{c}\right)\right]\left[1-\frac{1}{c}\left(\frac{\partial \mathbf{x}_{\mathrm{Ea}}\left(t_{t}\right)}{\partial t_{t}} \cdot \hat{\mathbf{r}}_{u p}\right)\right]^{-1}
\end{gathered}
$$

All the above computations are in SSB with TDB; however, the frequency measurements, at both $t_{t}$ and $t_{r}$, are done on Earth, that is with a time which is 
TT. This introduces a change in the measured frequencies at both ends, and because this change is not the same (the Earth having moved by about $3 \times 10^{-4}$ of its orbit) there is a correction needed to be performed. The quantity we are measuring is essentially the derivative of $t_{t}$ with respect to $t_{r}$, but in two different time systems (for readability, we use $T$ for TT, $t$ for TDB):

$$
\frac{d T_{t}}{d T_{r}}=\frac{d T_{t}}{d t_{t}} \frac{d t_{t}}{d t_{r}} \frac{d t_{r}}{d T_{r}}
$$

where the derivatives of the time coordinate changes are the same as the right hand side of the differential equation giving $T$ as a function of $t$ in the first factor and the inverse of the same for the last factor. However, the accuracy required is such that the main term with the gravitational mass of the Sun $\mu_{0}$ and the position of the Sun $\mathbf{x}_{0}$ is enough:

$$
\begin{aligned}
\frac{d T_{t}}{d T_{r}}= & {\left[1-\frac{\mu_{0}}{\left|\mathbf{x}_{E}\left(t_{t}\right)-\mathbf{x}_{0}\left(t_{t}\right)\right| c^{2}}-\frac{1}{2 c^{2}}\left|\frac{d \mathbf{x}_{\mathrm{E}}\left(t_{t}\right)}{d t_{t}}\right|^{2}\right] \frac{d t_{t}}{d t_{r}} } \\
& {\left[1-\frac{\mu_{0}}{\left|\mathbf{x}_{\mathrm{E}}\left(t_{r}\right)-\mathbf{x}_{0}\left(t_{r}\right)\right| c^{2}}-\frac{1}{2 c^{2}}\left|\frac{d \mathbf{x}_{\mathrm{E}}\left(t_{r}\right)}{d t_{r}}\right|^{2}\right]^{-1} . }
\end{aligned}
$$

Note that we do not need the $L_{C}$ constant term discussed in Section 2 because it cancels in the first and last term in the right hand side of (9). The correction of the above formula is required for consistency, but the correction has an order of magnitude of $10^{-7} \mathrm{~cm} / \mathrm{s}$ and is negligible for the sensitivity of the BepiColombo Radio Science Experiment (Figure 5).

\section{Numerical problems and solutions}

The computation of the observables, as presented in the previous section, is already complex, but still the list of subtle technicalities is not complete. A problem well known in radio science is that, for top accuracy, the range rate measurement cannot be the value $d r\left(t_{r}\right) / d t_{r}=\left(d r_{d o}\left(t_{r}\right) / d t_{r}+d S_{d o} / d t_{r}+d r_{u p}\left(t_{r}\right) / d t_{r}+\right.$ $\left.d S_{u p} / d t_{r}\right) / 2$. In fact, the measurement is not instantaneous: an accurate measure of a Doppler effect requires to fit the difference of phase between carrier waves, the one generated at the station and the one returned from space, accumulated over some integration time $\Delta$, typically between 10 and 1000 s. Thus the observable is really a difference of ranges

$$
\frac{r\left(t_{b}+\Delta / 2\right)-r\left(t_{b}-\Delta / 2\right)}{\Delta}
$$

or, equivalently, an averaged value of range rate over the integration interval

$$
\frac{1}{\Delta} \int_{t_{b}-\Delta / 2}^{t_{b}+\Delta / 2} \frac{d r(s)}{d t_{r}} d s .
$$


In order to understand the computational difficulty we need to take also into account the orders of magnitude. As said in the introduction, for state of the art of tracking systems, such as those using a multi-frequency link in the $\mathrm{X}$ and Ka bands, the accuracy of the range measurements can be $\simeq 10 \mathrm{~cm}$ and the one of range-rate $3 \times 10^{-4} \mathrm{~cm} / \mathrm{s}$ (over an integration time of $1000 \mathrm{~s}$ ). Let us take an integration time $\Delta=30 \mathrm{~s}$, which is adequate for measuring the gravity field of Mercury: in fact, if the orbital period of the spacecraft around Mercury is $\simeq 8000 \mathrm{~s}$, the harmonics of order $m=26$ have periods as short as $\simeq 150$ $\mathrm{s}$. The accuracy over $30 \mathrm{~s}$ of the range rate measurement can be, by Gaussian statistics, $\simeq 3 \times 10^{-4} \sqrt{1000 / 30} \simeq 17 \times 10^{-4} \mathrm{~cm} / \mathrm{s}$, and the required accuracy in the computation of the difference $r\left(t_{b}+\Delta / 2\right)-r\left(t_{b}-\Delta / 2\right)$ is $\simeq 0.05 \mathrm{~cm}$. The distances can be as large as $\simeq 2 \times 10^{13} \mathrm{~cm}$, thus the relative accuracy in the difference needs to be $2.5 \times 10^{-15}$. This implies that rounding off is a problem with current computers, with relative rounding off error of $\varepsilon=2^{-52}=2.2 \times 10^{-16}$ (Figure 6); extended precision is supported in software, but it has many limitations. The practical consequences are that the computer program processing the tracking observables, at this level of precision and over interplanetary distances, needs to be a mixture of ordinary and extended precision variables. Any imperfection may result in "banding", that is residuals showing a discrete set of values, implying that some information corresponding to the real accuracy of the measurements has been lost in the digital processing.
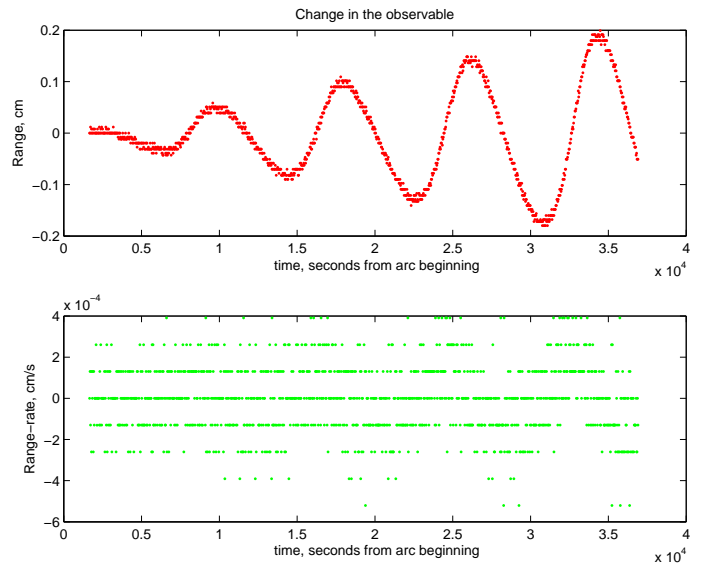

Figure 6: Range and range rate differences due to a change by $10^{-11}$ of the $C_{22}$ harmonic coefficient: the range rate computed as range difference divided by the integration time of 30 $\mathrm{s}$ is obscured by the rounding off.

As an alternative, the use of a quadrature formula for the integral in (11) can provide a numerically more stable result, because the $\mathrm{S} / \mathrm{N}$ of the range rate measurement is $\ll 1 / \varepsilon$. Figure 7 shows that a very small model change, generating a range rate signal $\leq 2 \mathrm{micron} / \mathrm{s}$ over one pass, can be computed smoothly by using a 7 nodes Gauss quadrature formula. 

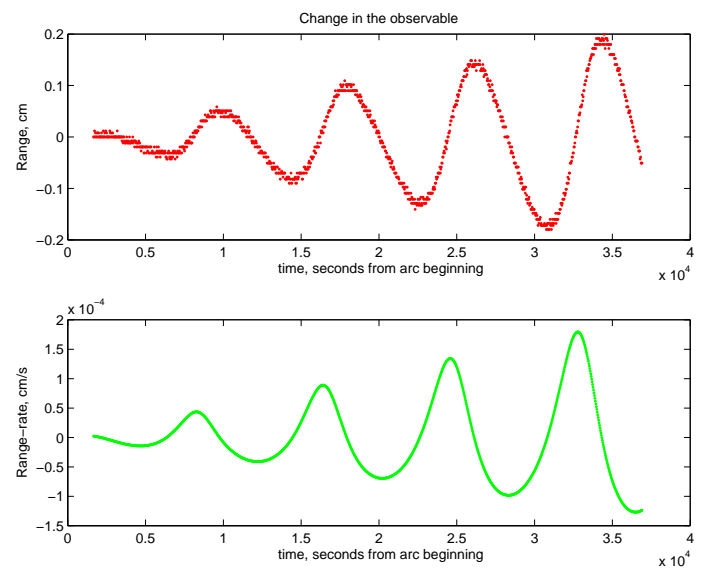

Figure 7: Range and range rate differences due to a change by $10^{-11}$ of the $C_{22}$ harmonic coefficient: the range rate computed as an integral is smooth; the difference is marginally significant with respect to the measurement accuracy.

\section{Conclusions}

By combining the results of (Milani et al. 2010) and of this paper, we have completed the task of showing that it is possible to build a consistent relativistic model of the dynamics and of the observations for a Mercury orbiter tracked from the Earth, at a level of accuracy and self-consistency compatible with the very demanding requirements of the BepiColombo Radio Science Experiment. In particular, in this paper we have defined the algorithms for the computation of the observables range and range rate, including the reference system effects and the Shapiro effect. We have shown which computations can be performed explicitly and which ones need to be obtained from an iterative procedure. We have also shown how to push these computations, when implemented in a realistic computer with rounding-off, to the needed accuracy level, even without the cumbersome usage of quadruple precision. The list of "relativistic corrections", assuming that we can distinguish their effects separately, is long, and we have shown that many subtle effects are relevant to the required accuracy. However, in the end what is required is just to be fully consistent with a post-Newtonian formulation to some order, to be adjusted when necessary. Interestingly, the high accuracy of BepiColombo radio system may require implementation of the second post-Newtonian effects in range.

Acknowledgements The authors thank the anonymous reviewer for his constructive comments useful to improve the presentation of the work. The results of the research presented in this paper, as well as in the previous one (Milani et al. 2010), have been performed within the scope of the contract ASI/2007/I/082/06/0 with the Italian Space Agency. BepiColombo is a scientific space mission of the Science Directorate of the European Space Agency. The work of DV was partially supported by the 
Czech Grant Agency (grant 202/09/0772) and the Research Program MSM0021620860 of the Czech Ministry of Education.

\section{References}

[Ashby and Bertotti 2009] Ashby, N., Bertotti, B.: Accurate light-time correction due to a gravitating mass, ArXiv e-prints, 0912.2705 (2009)

[Bertotti et al. 2003] Bertotti, B., Iess, L., Tortora, P.: A test of general relativity using radio links with the Cassini spacecraft. Nature, 425, 374-376 (2003)

[Damour et al. 1994] Damour, T., Soffel, M., Hu, C.: General-relativistic celestial mechanics. IV. Theory of satellite motion. Phys. Rev. D, 49, 618-635 (1994)

[Iess and Boscagli 2001] Iess, L., Boscagli, G.: Advanced radio science instrumentation for the mission BepiColombo to Mercury. Plan. Sp. Sci., 49, 1597-1608 (2001)

[Klioner and Zschocke 2007] Klioner, S.A., Zschocke, S.: GAIA-CA-TN-LOSK-002-1 report (2007)

[Klioner 2008] Klioner, S.A.: Relativistic scaling of astronomical quantities and the system of astronomical units. Astron. Astrophys., 478, 951-958 (2008)

[Klioner et al. 2010] Klioner, S.A., Capitaine, N., Folkner, W., Guinot, B., Huang, T. Y., Kopeikin, S., Petit, G., Pitjeva, E., Seidelmann, P. K., Soffel, M.: Units of Relativistic Time Scales and Associated Quantities. In: Klioner, S., Seidelmann, P.K., Soffel, M. (eds.) Relativity in Fundamental Astronomy: Dynamics, Reference Frames, and Data Analysis, IAU Symposium, 261, 79-84 (2010)

[Milani et al. 2002] Milani, A., Vokrouhlický, D., Villani, D., Bonanno, C., Rossi, A.: Testing general relativity with the BepiColombo radio science experiment. Phys. Rev. D, 66, 082001 (2002)

[Milani and Gronchi 2010] Milani A., Gronchi G.F.: Theory of orbit determination. Cambridge University Press (2010)

[Milani et al. 2010] Milani, A., Tommei, G., Vokrouhlický, D., Latorre, E., Cicalò, S.: Relativistic models for the BepiColombo radioscience experiment. In: Klioner, S., Seidelmann, P.K., Soffel, M. (eds.) Relativity in Fundamental Astronomy: Dynamics, Reference Frames, and Data Analysis, IAU Symposium, 261, 356-365 (2010)

[Moyer 2003] Moyer, T.D.: Formulation for Observed and Computed Values of Deep Space Network Data Types for Navigation. Wiley-Interscience (2003) 
[Shapiro 1964] Shapiro, I.I.: Fourth test of general relativity. Phys. Rev. Lett., 13, 789-791 (1964)

[Soffel et al. 2003] Soffel, M., Klioner, S.A., Petit, G., Kopeikin, S.M., Bretagnon, P., Brumberg, V.A., Capitaine, N., Damour, T., Fukushima, T., Guinot, B., Huang, T.-Y., Lindegren, L., Ma, C., Nordtvedt, K., Ries, J.C., Seidelmann, P.K., Vokrouhlický, D., Will, C.M., Xu, C.: The IAU 2000 resolutions for astrometry, celestial mechanics, and metrology in the relativistic framework: explanatory supplement. Astron. J., 126, 2687-2706 (2003)

[Teyssandier and Le Poncin-Lafitte 2008] Teyssandier, P., Le Poncin-Lafitte, C.: General post-Minkowskian expansion of time transfer functions. Class. Quantum Grav., 25, 145020 (2008)

[Will 1993] Will, C.M.: Theory and experiment in gravitational physics. Cambridge University Press (1993)

[Yeomans et al. 1992] Yeomans, D. K., Chodas, P. W., Keesey, M. S., Ostro, S. J., Chandler, J. F., Shapiro, I. I.: Asteroid and comet orbits using radar data. Astron. J., 103, 303-317 (1992) 